\title{
Effect of indium content on performance and reliability of InGaN/GaN light- emitting diodes
}

\author{
Z. L. Li, ${ }^{1}$ S. Tripathy, ${ }^{2}$ P. T. Lai, ${ }^{1}$ and H. W. Choi ${ }^{1, a)}$ \\ ${ }^{1}$ Department of Electrical and Electronic Engineering, The University of Hong Kong, Hong Kong \\ ${ }^{2}$ Institute of Materials Research and Engineering, A*STAR (Agency for Science, Technology, and Research), \\ 3 Research Link, Singapore 117602
}

(Received 3 July 2009; accepted 29 September 2009; published online 10 November 2009)

\begin{abstract}
While longer wavelength emission from InGaN/GaN light-emitting diodes (LEDs) can be achieved by increasing the indium (In) content in the quantum wells, the increased In content gives rise to side effects to the material and device performance and reliability. It was found that the induced strain in the wafer and the density of threading dislocations increase with increasing In content. From current-voltage and $1 / f$ noise measurements, it was observed that the leakage currents, static resistance, and noise magnitudes rise monotonically with increasing emission wavelength (In composition), which can be attributed to higher defect concentrations. After undergoing a $1000 \mathrm{~h}$ reliability test, it was discovered that the optical degradation rates for the longer wavelength green LEDs were significantly higher than those of shorter wavelength. (C) 2009 American Institute of Physics. [doi:10.1063/1.3253754]
\end{abstract}

\section{INTRODUCTION}

The role of varying indium molar fractions in InGaN/ GaN heterostructures on its optical properties has been extensively reported. ${ }^{1}$ In a light-emitting diode (LED) structure, the indium concentration in the InGaN/GaN multiquantum wells (MQWs) is varied in order to produce LEDs with different emission wavelengths across the visible spectrum. However, with increasing indium content to produce LEDs of longer wavelengths, the strain at the interface of $\mathrm{InGaN}$ and $\mathrm{GaN}$ increases correspondingly, leading to a higher compressive strain in the well layer, resulting in the difficulty of effective indium incorporation. ${ }^{2}$

The implication of having a higher indium ratio in the MQWs is its potential threat to device performance and longer-term reliability. First, the presence of higher In content will introduce large piezoelectric fields induced by the large strain. This piezoelectric field reduces the overlap of the electron-hole wave functions, and so results in reduced internal quantum efficiencies in longer-wavelength LEDs. ${ }^{3}$ Second, the large built-in strain can give rise to high defect concentrations in the structure in the form of dislocations, which results in a degraded material quality. As a result, the relatively higher dislocation densities of longer-wavelength green LEDs often lead to poorer optical performance and compromised reliability.

Our study is divided into two parts. First, we perform material characterizations on InGaN/GaN LED wafers of different In content (and thus emission wavelengths). Atomic force microscopy (AFM) surface scans were used to detect the etch pits in plasma-etched wafer surfaces; etch pits offer an accurate indication of defect densities in $\mathrm{GaN}$ wafers. Raman spectroscopy was employed to evaluate the strain condition in the QW regions of wafers. Additionally, the Hall

\footnotetext{
${ }^{\text {a) }}$ Author to whom correspondence should be addressed. Electronic addresses: hwchoi@hku.hk and hwchoi@eee.hku.hk.
}

mobility was measured, which were subsequently used for explaining the acquired noise spectra of devices.

The second part relates to device characterization. LEDs of wavelengths ranging from 440 to $550 \mathrm{~nm}$ were fabricated under identical process conditions and device geometries. The device performance and reliability were evaluated by correlating defect concentrations in the various wafers with their respective low frequency noise spectrum and $1000 \mathrm{~h}$ degradation curve. The electrical properties of the different devices, including $I-V$ characteristics, leakage current, $1 / f$ noise spectrum were measured. The devices were subsequently subjected to a $1000 \mathrm{~h}$ continuous stress test. The results serve to prove our postulation, and a physical model has been constructed to explain the observed phenomenon.

\section{EXPERIMENTAL DETAILS}

The general structure of the LED wafers consist of a GaN buffer layer grown on $c$-plane sapphire substrate, followed by a thick $n$-type GaN layer, active layers with InGaN/GaN quantum wells, and capped with a top $p$-type $\mathrm{GaN}$ contact layer. The nominal center wavelengths of the grown wafers are 440, 470, 520, 540, and $550 \mathrm{~nm}$ achieved by varying the alloying ratio of $\operatorname{In}_{x} \mathrm{Ga}_{1-x} \mathrm{~N}$ from $x=0.15$ to 0.31 , and are labeled samples A-E in the order of increasing wavelength. Details of the growth will be reported elsewhere. The defect densities of the grown wafers were determined by AFM using a Seiko Instruments Nanopics system, after defect exposure by a plasma treatment similar to the mesa etch process to be described later. UV-Raman spectroscopy uses a $325 \mathrm{~nm} \mathrm{He}-\mathrm{Cd}$ laser as an excitation source focused to a spot of less than $1 \mu \mathrm{m}$. The scattered light was detected in the backscattering configuration with a JobinYvon T64000 triple-grating spectrometer with a $\mathrm{LN}_{2}$-cooled charge coupled device detector. The hole mobility was characterized by a Biorad HL5500 Hall effect measurement system in the Van Der Pauw configuration. 


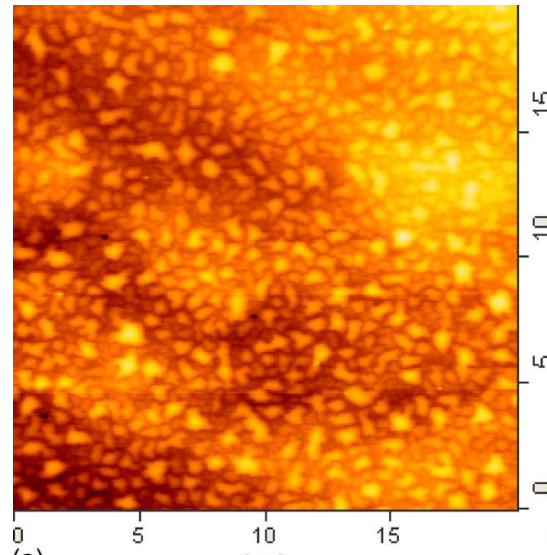

(a)

\section{[нтा]}

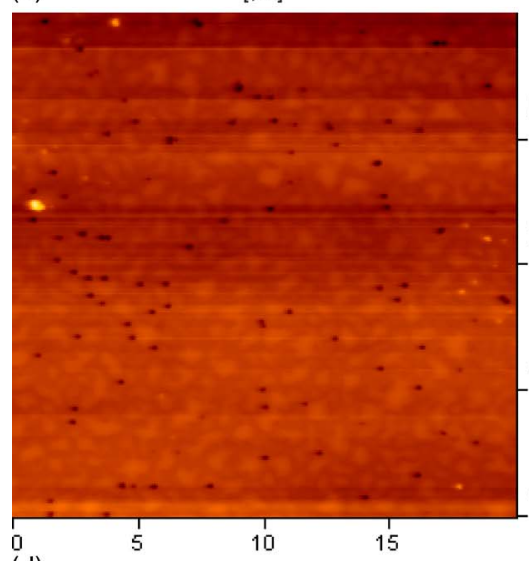

(d)

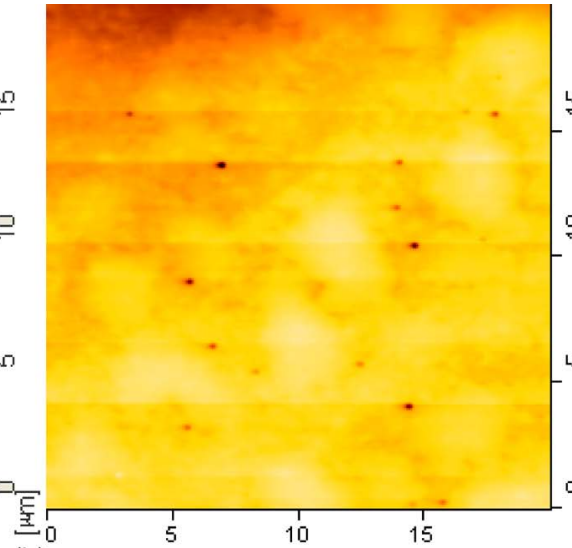

(b)
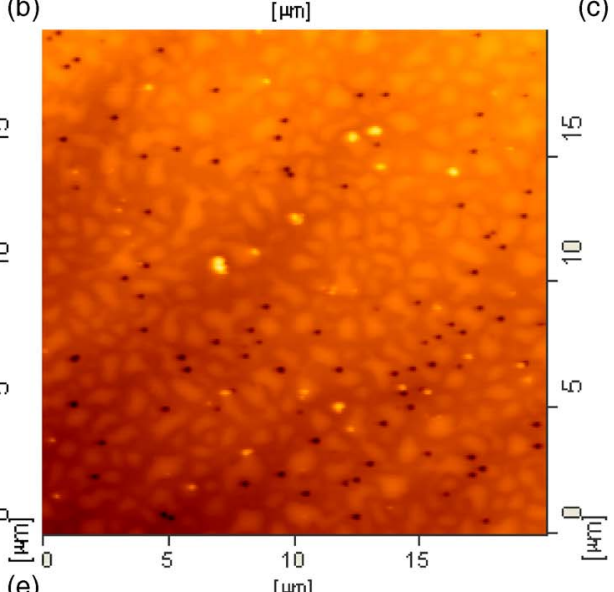

(e) [нтा]

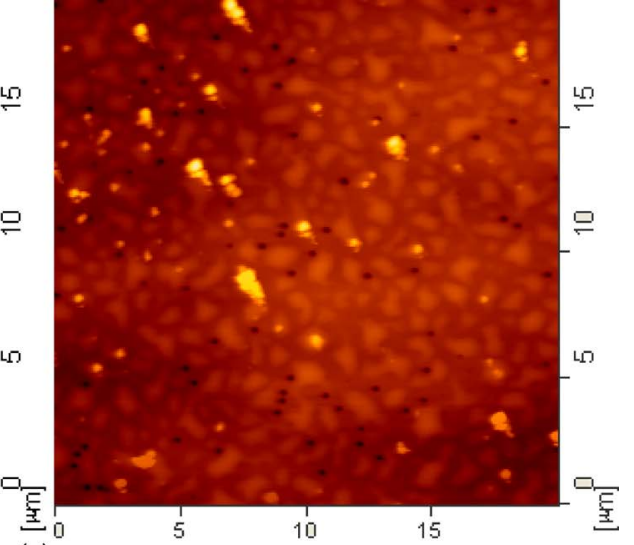

(c) [нтा]

FIG. 1. (Color online) $20 \times 20 \mu \mathrm{m}$ AFM surface scan of the (a) $440 \mathrm{~nm}$, (b) $470 \mathrm{~nm}$, (c) $520 \mathrm{~nm}$, (d) $540 \mathrm{~nm}$, and (e) $550 \mathrm{~nm} \mathrm{InGaN/GaN} \mathrm{LED} \mathrm{wafers.}$

The LEDs were fabricated via a standard microfabrication process beginning with the deposition of a semitransparent current-spreading layer composed of $\mathrm{Ni} / \mathrm{Au}(10 \mathrm{~nm} / 10$ $\mathrm{nm}$ ) by e-beam evaporation, followed by annealing in an $\mathrm{O}_{2}$ environment at a temperature of $550{ }^{\circ} \mathrm{C}$ for $5 \mathrm{~min}$. A 500 $\times 500 \mu \mathrm{m}^{2}$ emission active area was defined by photolithography and dry etched using a chemistry comprising $\mathrm{CHF}_{3}$ and Ar to expose the $n$-contact regions. Photolithography was carried out again to define the $p$-pad and $n$-pad areas. A bilayer of Ti/Al $(50 \mathrm{~nm} / 350 \mathrm{~nm})$ was deposited as $p$-pads and $n$-pads, and then annealed in $\mathrm{N}_{2}$ at $350{ }^{\circ} \mathrm{C}$ for 1 min. The chips were diced by laser micromachining and packaged onto TO headers. After wire bonding, a silicone encapsulant was applied to protect the devices. The electrical properties, including the $I-V$ characteristics and leakage current of the packaged devices were measured with a HP 4156A precision semiconductor parameter analyzer, while the $1 / f$ noise spectrum was measured by a BTA 9603 FET Noise Analyzer. The optical performance of the devices was evaluated with an Ocean Optics HR2000 fiber-coupled spectrometer. The devices, biased at $20 \mathrm{~mA}$, were subsequently subjected to a $1000 \mathrm{~h}$ continuous stress test at room temperature.

\section{RESULTS AND DISCUSSION}

\section{A. Material characterization}

The AFM surface scans in Fig. 1 are used for defect estimation of the LED wafers. Defects propagate from the site where they are generated, forming line dislocations. These defects include those generated at the GaN/sapphire interface due to lattice mismatch and also defects generated within the MQWs due to strain. Since dislocations terminate at an interface as voids, counting the number of such voids within unit area provides data on the dislocation density. Nevertheless, such voids are of subnanometer dimension. In order to "visualize" these features, they can be enlarged by a plasma treatment. By etching away $\sim 50 \mathrm{~nm}$ of $p$-type $\mathrm{GaN}$ from the surface of the wafer, the defects are intentionally enlarged due to preferential etching of recessed areas, ${ }^{4}$ resulting in the formation of etch pits. Etch pits are readily revealed by AFM scanning of the plasma-treated surfaces, from which the threading dislocation density can be estimated by counting the number of spots within a known area. In the $20 \times 20 \mu \mathrm{m}^{2}$ surface scans shown in Fig. 1, the dark dots represent these etch pits. The estimated defect densities for sampled A-E are listed in Table I, respectively. Of course, these figures do not represent the actual dislocation densities in the wafer, as a portion of the dislocations are annihilated at interfaces. ${ }^{5}$ Nevertheless, these numbers serve to provide a comparative indication of the material quality. For example, an order of magnitude increase in etch pits is observed between wafers $\mathrm{A}$ and $\mathrm{B}$ when the In content was raised from $\sim 0.15$ to $\sim 0.20$, and also between wafers $B$ and $\mathrm{C}$ with a similar fractional change in In content. A significant increase in defect density is positively identified for wafers of increasing wavelengths. 
TABLE I. Experimental data obtained from the five wafers, including etch pit density, $E_{2}$ phonon frequency, Hall mobility, and device static resistance.

\begin{tabular}{lcccc}
\hline \hline $\begin{array}{l}\text { Sample/ } \\
\text { Device }\end{array}$ & $\begin{array}{c}\text { Etch pit } \\
\text { density/cm }\end{array}$ & $\begin{array}{c}E_{2} \text { phonon } \\
\text { frequency/cm }\end{array}$ & $\begin{array}{c}\text { Hall mobility/ } \\
\mathrm{cm}^{2} \mathrm{~V}^{-1} \mathrm{~s}^{-1}\end{array}$ & $\begin{array}{c}\text { Static } \\
\text { resistance/ } \Omega\end{array}$ \\
\hline A-440 nm & $7.5 \times 10^{5}$ & 570.0 & 8.56 & 13.9 \\
$\mathrm{~B}-470 \mathrm{~nm}$ & $3.0 \times 10^{6}$ & 570.6 & 3.36 & 14.2 \\
$\mathrm{C}-520 \mathrm{~nm}$ & $1.4 \times 10^{7}$ & 570.5 & $\ldots$ & 23.5 \\
D-540 nm & $1.75 \times 10^{7}$ & 571.2 & 2.50 & 33.3 \\
E-550 nm & $2.22 \times 10^{7}$ & 572.2 & 2.78 & 52.6 \\
\hline \hline
\end{tabular}

Strain in GaN epitaxial layers originates from growth on the lattice-mismatched sapphire substrate and subsequent postgrowth cooling, and also due to the presence of point defects, leading to the formation of threading dislocations. ${ }^{6}$ The $E_{2}$ mode frequency from a Raman spectrum can effectively be used to monitor the strain component in the structure, since it is much more sensitive to strain compared to other Raman modes due to its independence from the free carrier concentration. The $E_{2}$ phonon line was reported to shift toward the higher-frequency region with increasing compressive strain by $\sim 2.8 \mathrm{~cm}^{-1} / \mathrm{GPa}^{7}$ Since we are investigating the effects of varying In content in the quantum wells, UV-Raman spectroscopy was conducted to ensure that the probe depth was shallow. The Raman spectra taken from six wafers of different wavelengths are shown in Fig. 2.

By performing a Lorentzian fit to spectra the center value of the $E_{2}$ phonon peak can be determined, which have been listed in Table I. For comparison, the $E_{2}$ mode center frequency of $567.3 \mathrm{~cm}^{-1}$ was measured from a piece of free standing GaN, corresponding to nearly strain-free $\mathrm{GaN}{ }^{8} \mathrm{~A}$ monotonic linear shift of phonon frequency was observed with increasing wavelength, highlighting the role of In doping in stressing the quantum well regions.

The presence of dislocation can affect carrier scattering in a material. ${ }^{9}$ In this respect, the Hall mobility of our series of samples has been measured. It is desirable to measure the Hall mobility of the $p$-doped layer only. To prevent current penetration across the $p-n$ junction and parallel conduction

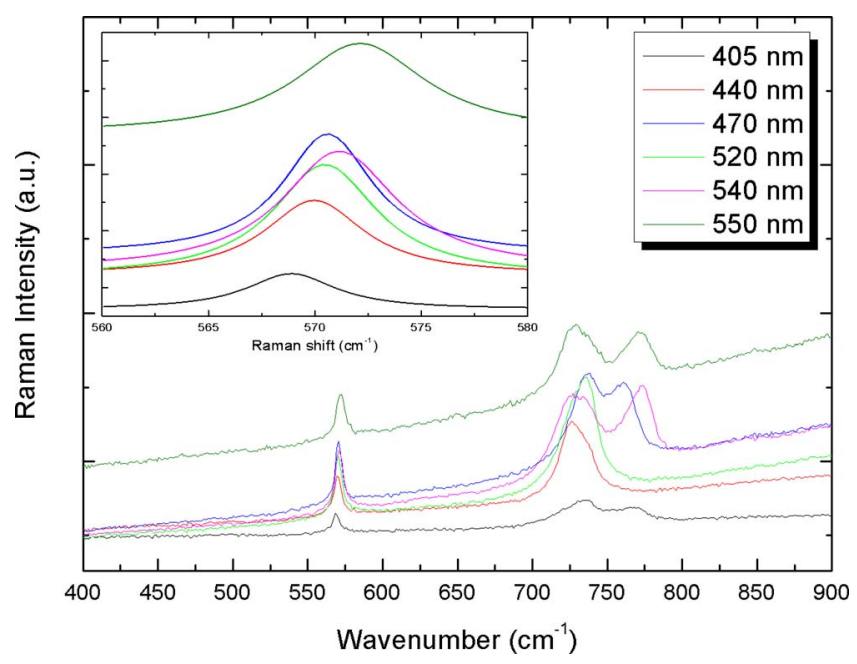

FIG. 2. (Color online) UV-Raman spectra for wafers of wavelengths ranging from 405 to $550 \mathrm{~nm}$.

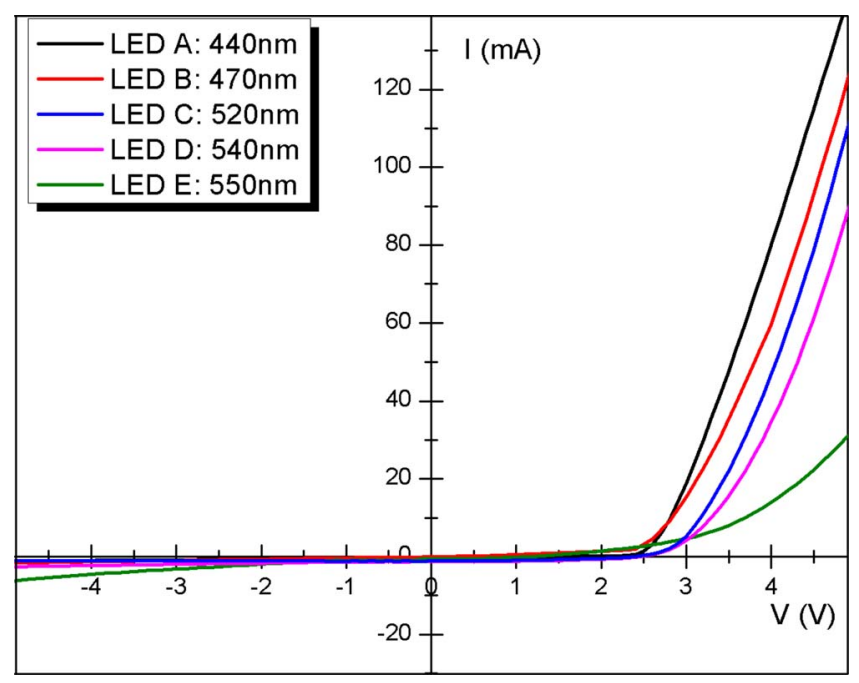

FIG. 3. (Color online) $I-V$ characteristics of the five InGaN/GaN LEDs of different emission wavelengths.

along different layers, the test current was limited to $0.5 \mathrm{~mA}$. The measured Hall mobilities are listed in Table I.

From the Hall measurement result shown in Table I, the hole mobility decreases from $8.56 \mathrm{~cm}^{2} \mathrm{~V}^{-1} \mathrm{~s}^{-1}$ in wafer A to $2.78 \mathrm{~cm}^{2} \mathrm{~V}^{-1} \mathrm{~s}^{-1}$ in wafer $\mathrm{E}$, a result very likely to be due to dislocation scattering. Since carrier drift motion is mostly restricted by traps and defects in the material and that the $p$-dopant concentration is identical across different wafers, the result suggests that the lower mobilities in the longerwavelength wafers are caused by carrier scattering at the site of dislocations..

\section{B. Device characterization}

The electrical characteristics of the LED devices fabricated wafers A-E are evaluated. For consistency, these devices are labeled $\mathrm{A}-\mathrm{E}$ in the order of increasing wavelength. The current-voltage $(I-V)$ characteristics of the five LEDs are presented in Fig. 3. The leakage currents of the devices have been extracted at a reverse bias voltage of $4 \mathrm{~V}$. As the emission wavelength of the LEDs increases, the leakage currents also increase from $0.95 \mathrm{~mA}$ in device A to $0.98,0.99,2.29$, and $4.45 \mathrm{~mA}$ in devices $\mathrm{B}, \mathrm{C}, \mathrm{D}$, and $\mathrm{E}$, respectively. The slope of the linear region of the $I-V$ curves (in the forward bias region) has been interpolated to determine the static resistance of the devices. As evident from the $I-V$ plot, the slope increases as the wavelength increases. The extracted static resistances are 13.9, 14.2, 23.5, 33.3, and $52.6 \Omega$ for devices A-E, respectively. Such monotonic increase in leakage current and static resistance can be linked to the increasingly higher defect concentration as the In incorporation increases. The dislocations act as vertical current leakage pathways, while the defects lead to carrier scattering and thus higher static resistance and reduced carrier mobility. To verify these further, noise measurements were conducted to study the carrier properties.

Low-frequency noise measurement is widely recognized as an effective tool for characterization of the quality of a material. Compared to other reliability characterization methods, noise measurement is more sensitive and contains 


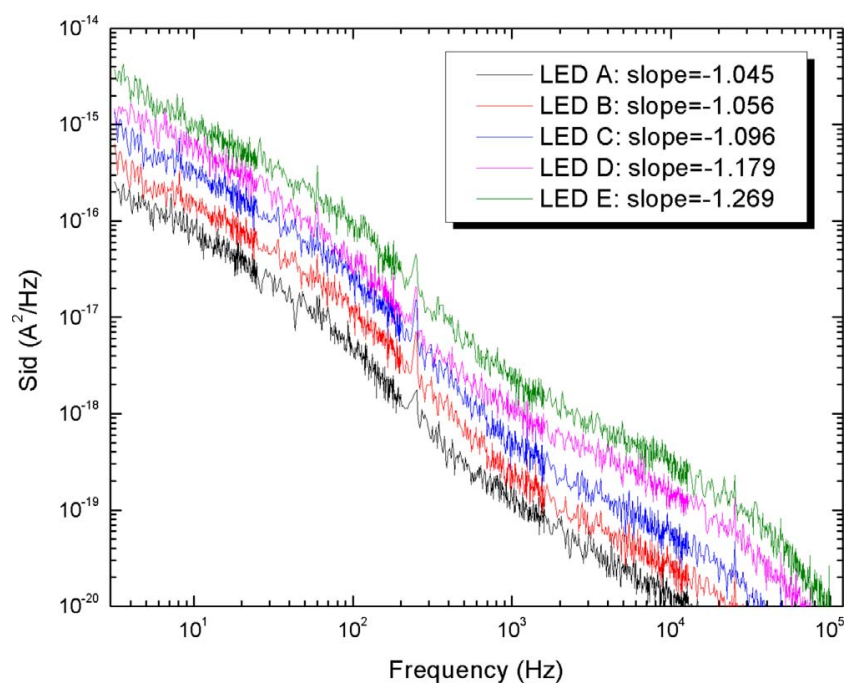

FIG. 4. (Color online) Noise spectrum of LEDs A-E.

richer information pertaining to device inner quality. ${ }^{10,11}$ Many researches indicate that low-frequency noise is closely associated with the impurities and defects in the devices, such as traps in oxide layer and dislocations in crystal and interface states. ${ }^{12}$ Low-frequency noises, especially $1 / f$ noise and generation-recombination noise, are thus deemed important factors for determining the reliability of a LED. Since low frequency noise is closely related to the presence of impurities and defects in the semiconductors, it can accurately represent the internal crystal quality. ${ }^{13-16}$ The noise spectrums of the five LEDs were measured in a shielded environment and are plotted in Fig. 4. The system floor calibration was conducted for dc bias calculation and noise correction. The range of frequencies was between $3.15 \mathrm{~Hz}$ and $105 \mathrm{kHz}$, and the measurements were carried out at a constant bias current of $10 \mathrm{~mA}$. The slope can be extracted by the supplied software automatically. From the figure, both the magnitude and slope demonstrate a consistent trend with increasing wavelength.

In an LED or any $p$ - $n$ junction diode structure, the current spectral density under low current bias can be expressed by the following equation: ${ }^{14}$

$$
S_{I}=\frac{4 N_{t} q I_{D}}{\tau_{r} \sigma \nu N_{c}^{2}} e^{2 E_{t} / k T},
$$

where $N_{t}$ is the trap density; a larger $N_{t}$ value generally implies poorer crystal quality and increases the magnitude of the noise spectrum. $I_{D}$ is the diode current through the device, $\sigma$ the capture cross section, $\nu$ the thermal velocity determined by the temperature, $N_{c}$ the effective density of states in the conduction band, and $E_{t}$ the level position of trap. Also, $\tau_{r}$ is the recombination time or carrier lifetime, which can increase the magnitude of the noise spectrum when the trap density increases, while the other terms have their usual meanings.

The measured noise spectrum thus indicates that the crystal quality deteriorates as the emission wavelength increases from 440 to $550 \mathrm{~nm}$ due to the increased noise magnitude, supporting the observation of increased defect density with increasing In incorporation. Apart from the noise

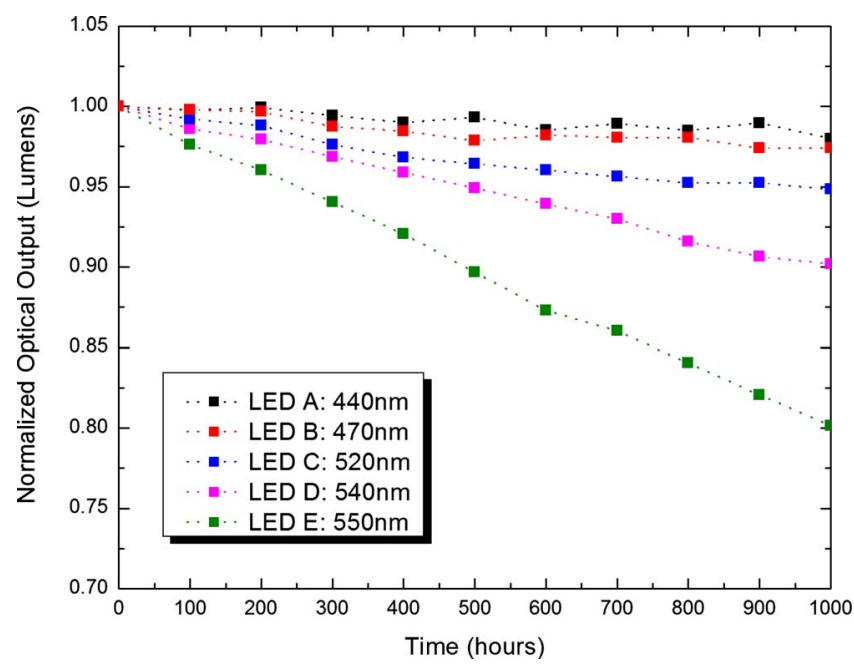

FIG. 5. (Color online) $1000 \mathrm{~h}$ lifetime degradation test of the five LEDs.

magnitude, it is also observed that the magnitude of the slope of the noise spectrum increases with increasing wavelength, which has been indicated in the legend of Fig. 4. This may be explained in terms of occupation of traps within the semiconductor material. As defect density in the InGaN/GaN layers increases, transitions between traps become more frequent, leading to a reduction in electron mean free time. This can be deduced from the carrier mobility obtained from Hall measurement. The hole mobility in the layer $p$-GaN decreases with increasing emission wavelength of the wafer, which we attributed to increased dislocation densities. These defects actively trap/detrap the carriers, and also act as scattering centers. Thus, both the majority and minority carrier lifetimes will decrease in a wafer with higher defect density. According to Eq. (1), the decreased minority lifetime will increase the magnitude of noise spectrum. Therefore, as the frequency increases, these trapped electrons cannot respond quickly enough and the number of free electron in the crystal decreases, which subsequently leads to a decrease in the current spectral density at that particular frequency. With more defects in the crystal, the spectrum decreases at a faster rate, corresponding to an increase in the slope, which is consistent with our measured data.

The rate at which optical output power degrades is a direct manifest of device reliability. Figure 5 shows the results of a $1000 \mathrm{~h}$ reliability test, whereby a constant bias current of $20 \mathrm{~mA}$ was applied to the devices. While the 440 nm LED-A maintain $\sim 98 \%$ of its initial optical power after $1000 \mathrm{~h}$, the $470 \mathrm{~nm}$ LED-B maintains a similar level of 97\% of its initial optical power, and the $520 \mathrm{~nm}$ LED-C maintains $\sim 95 \%$ of its initial optical power. This percentage drops to $\sim 91 \%$ and $\sim 82 \%$ for the 540 and $550 \mathrm{~nm}$ LEDs, respectively. Assuming linearity, the L70 lifetime [L70 lifetime is the time to $70 \%$ lumen maintenance, as proposed by ASSIST (Ref. 17)] for LEDs A-E are 15 000, 10 000, 6000, 3750, and $1667 \mathrm{~h}$, respectively. LEDs with increasing wavelength are therefore expected to have increasingly shorter lifetime.

Increasing In concentration is essential to achieving longer wavelength emission from $\mathrm{InGaN} / \mathrm{GaN}$ materials. Theoretically, the bandgap of this material system can be 
tuned from $\sim 360 \mathrm{~nm}$ (for $\mathrm{GaN}$ ) to $\sim 650 \mathrm{~nm}$ (for $\mathrm{InN}$ ). Intuitively, the monolithic integration of MQWs emitting at the primary colors is possible, producing a single-chip white light LED without external color-conversion materials such as phosphors. However, our study reveals the difficulty of increasing In concentration beyond a certain threshold. As a matter of fact, blue $(\sim 460 \mathrm{~nm})$ and green $(\sim 520 \mathrm{~nm})$ InGaN/GaN LEDs are most commonly available in the market. Devices emitting at longer wavelengths are restricted to laboratory demonstrations. Based on current epitaxial technology, knowledge of the material system, and availability of substrates, it is not feasible to grow InGaN/GaN MQWs with emission beyond $\sim 540 \mathrm{~nm}$ as the defect density rises drastically, degrading optical and electrical performance, and also long-term reliability. The AlInGaP material system is definitely more mature for that wavelength range. Nevertheless, the target of achieving high performance long wavelength emission from InGaN/GaN materials may become a reality with further improvements in material growth, better understanding of materials physics, and the availability of lattice-matched substrates. This is particularly so with advancements in $\mathrm{GaN}$ bulk substrate technology.

\section{CONCLUSIONS}

In summary, the effects of varying In concentration to the optical and electrical properties if InGaN/GaN LEDs have been studied. From the large numbers of parameters obtained from the materials and devices, a conclusion can be drawn. The defect density in the material grows significantly with increasing In ratio, attributed to strain introduced into the wafer by the dopants. The defects act as nonradiative recombination centers as well as leakage pathways, resulting in degraded optical performance, increased resistance, and accelerated deterioration rates in the devices. While higher In ratio in the MQWs can produce LEDs with longer wavelength and higher photopic response $(\sim 555 \mathrm{~nm})$ and thus highly suited for solid-state lighting, significant progress in material epitaxy is needed to reduce defect densities in order to compete with AlInGaP materials in the wavelength range.

${ }^{1}$ T. Takeuchi, H. Takeuchi, S. Sota, H. Sakai, H. Amano, and I. Akasaki, Jpn. J. Appl. Phys., Part 2 36, L177 (1997).

${ }^{2}$ C. F. Huang, T. Y. Tang, J. J. Huang, W. Y. Shiao, C. C. Yang, C. W. Hsu, and L. C. Chen, Appl. Phys. Lett. 89, 051913 (2006).

${ }^{3}$ R. D. Dupuis, J. B. Limb, J. P. Liu, J. H. Ryou, C. Horne, and D. Yoo, Proc. SPIE 6894, 68941D 2008.

${ }^{4}$ H. W. Choi, C. Liu, M. G. Cheong, J. Zhang, and S. J. Chua, Appl. Phys. A: Mater. Sci. Process. 80, 405 (2005).

${ }^{5}$ S. Ruvimov, Z. Liliental-Weber, T. Suski, J. W. Ager III, J. Washburn, J. Krueger, C. Kisielowski, E. R. Weber, H. Amano, and I. Akasaki, Appl. Phys. Lett. 69, 990 (1996).

${ }^{6}$ C. Kisielowski, J. Kruger, S. Ruvimov, T. Suski, J. W. Ager III, E. Jones, Z. Liliental-Weber, M. Rubin, and E. R. Weber, Phys. Rev. B 54, 17745 (1996).

${ }^{7}$ F. Demangeot, J. Frandon, M. A. Renucci, O. Briot, B. Gil, and R. L. Aulumbard, Solid State Commun. 100, 207 (1996).

${ }^{8}$ H. W. Choi, C. W. Jeon, M. D. Dawson, P. R. Edwards, R. W. Martin, S. Tripathy, and S. J. Chua, J. Appl. Phys. 93, 5978 (2003).

${ }^{9}$ H. M. Ng, D. Doppalapudi, T. D. Moustakas, N. G. Weimann, and L. F. Eastman, Appl. Phys. Lett. 73, 821 (1998).

${ }^{10}$ Y. S. Dai and J. S. Xu, Solid-State Electron. 44, 1495 (2000).

${ }^{11}$ M. M. Jevti, Microelectron. Reliab. 35, 455 (1995).

${ }^{12}$ D. Ursutiu and B. K. Jones, Semicond. Sci. Technol. 11, 1133 (1996).

${ }^{13}$ F. N. Hooge, T. G. M. Kleinpenning, and L. K. J. Vandamme, Rep. Prog. Phys. 44, 479 (1981).

${ }^{14}$ S. Sawyer, S. L. Rumyantsev, M. S. Shur, N. Pala, Yu. Bilenko, J. P. Zhang, X. Hu, A. Lunev, J. Deng, and R. Gaska, J. Appl. Phys. 100, 034504 (2006).

${ }^{15}$ F. N. Hooge, IEEE Trans. Electron Devices 41, 1926 (1994).

${ }^{16}$ A. Balandin, K. L. Wang, A. Svizhenko, and S. Bandyopadhyay, IEEE Trans. Electron Devices 46, 1240 (1999).

${ }^{17}$ Alliance for Solid-state Illumination and Technologies; website: www.lrc.rpi.edu/programs/solidstate. 